\title{
GENERAL DISCUSSION
}

\section{Chairman: J. C. WATERLow, London School of Hygiene and Tropical Medicine, Keppel Street, London WC1E $7 H T$}

Chairman: Thank you very much both speakers. One of the problems is that many of us do not like being told by government what we ought to do. There are two people here in the audience, Dr Passmore and Miss Hollingsworth, who 7 years ago wrote an article called 'The better British diet'*. They were careful to avoid saying 'best'. In America they use the word 'prudent'. I like that because it leaves it open to us to be imprudent if we wish. It gives one freedom.

The point made about costs is very important. What league are we in as health professionals when it comes to costs? I have seen it estimated recently that the cost of saving a life in hospital by dialysis equipment, etc. is around $£ 100,000$ a life. The cost of saving a life by improving roads is one million pounds a life. The cost of saving a life by improving nuclear safety is 10 million pounds a life. That 10 million pounds will almost certainly be spent on nuclear safety. Will we be able to command those sums of money for nutrition? That is one of the things we have to think about.

Now I propose to call next on Professor Marmot to contribute perhaps in opposition to Professor Mitchell.

Professor M. Marmot: Thank you Mr Chairman. I will respond to some of the points raised by Professor Mitchell.

Professor Mitchell set up a dichotomy between the seeker of truth and the evangelist. Given that choice I would choose being a seeker of truth any time. This would automatically put me on Professor Mitchell's side, against the assembled evangelists here. I would also rather be on Professor Mitchell's side for two other reasons. First, it really is more fun poking holes in rather badly done trials, than trying to organize a better one. All studies have defects. Intellectually, you are in a sounder position looking at the evidence and criticizing it for its palpable shortcomings, than leaping for conclusions. Second, on Professor Mitchell's side, I will never have to eat my words. If one wants definitive proof before making any recommendations at all about dietary policy to prevent coronary disease, then of course the proof will never be forthcoming.

In addition, Professor Mitchell brings to the debate clarity, wit and stimulating, trenchant questions. Therefore, the only reason I am not on Professor Mitchell's side is I think he is wrong. The reason why I, if pushed, come down off the fence on one side is that I think the 'seeker of truth' position is likely to retard the progress of public health. The trials all have defects, but we must still make a decision about prevention of coronary heart disease on the face of those defects.

Professor Mitchell implied that we should not even try to prevent coronary heart disease because people may well die of something worse. Bearing this in mind, some of us still think that prevention of coronary heart disease is a worthwhile goal. Japan, which has the lowest coronary disease rate of the industrialized world, does in fact have the longest life expectancy of the industrialized world. Tony Mitchell said at the end, we should be considering not just mortality, but quality of life. If prevention of coronary

*Passmore, R., Hollingsworth, D. \& Robertson, J. (1979). British Medical Journal i, 527-531.

†Franklin. N. L. (1986). Science and Public Affairs 1, 23-41. 
heart disease actually prevents premature disability, for example cardiac neurosis, the depression that follows myocardial infarction, and the disabling that comes with angina pectoris and cardiac failure, then it seems reasonable that one should hope to do it. Tony Mitchell argues that even if we are successful we shall be left with old people with disabling problems. Will the measures that we take to prevent coronary heart disease lead to more or less disabling problems in old age? There is a whole debate in the field of geriatrics and ageing about the rectangular ageing curve, and whether or not the measures that you take to prevent disease may lead to a healthier old age. We do not know the answer to that question at this stage. The response to this uncertainty should not be to do nothing until we mount cohort studies and wait 50 years. I think we have to do something before that time and the question is what do we do?

You have heard about defects in trials of coronary heart disease prevention. There is a curious disparity in people's argument about whether medical practice should be based on evidence from trials. If a general practitioner does one thing a day that is based on hard scientific evidence that would be a special day in the week. In fact most of what doctors do is not based on evidence from trials. To illustrate: Professor Mitchell declared himself to be a passionate anti-smoker. If we look for the trial evidence that giving up smoking reduces total mortality, it does not exist. There is one trial of anti-smoking in primary prevention and that showed no impact on total mortality. It showed a reduction in lung cancer and an increase in non-lung cancers. There is no evidence based on trials that giving up smoking is good for you, if your outcome measure is total mortality. But I am as passionate an anti-smoker as Professor Mitchell. Why? Because we cannot make these decisions based on trial evidence. As Professor Mitchell pointed out in discussing the American MRFIT study, you cannot do these trials very easily. People change their behaviour in all sorts of uncontrolled ways (and one is delighted that that is the case).

In the American MRFIT trial, the net difference in plasma cholesterol between the intervention and the control group was $2 \%$. That is no test of a dietary hypothesis. It may be a test of people's ability to organize an effective trial but it is no test of a dietary hypothesis. I take the view that we cannot rely on trial data to make these decisions. Having said that, I am sure you are aware of Richard Peto's analysis of lumping together the trial data and coming up with a conclusion that supports the results of observational studies. Trials of cholesterol lowering, whether by drugs or diet, have led to a drop in myocardial infarction incidence, roughly proportionally to the drop in serum cholesterol. One does not need trials to establish the case for dietary prevention of coronary heart disease, but, in general, the trials do support the case.

There is not a neat, simple answer to this problem. Apart from the uncertainties in the dietary evidence, there is the influence of other causes, for example, smoking. We know that in this country, smoking is one of the major risk factors for coronary heart disease. Yet in Japan, smoking is totally unrelated, in the countries of Southern Europe smoking has a moderate relationship to coronary heart disease risk, but in this country, the United States and Northern Europe, smoking has a steep relationship to risk. It is a complex problem. One hypothesis as to what is different about Japan, Southern Europe, Northern Europe and the United States is the diet and the level of plasma lipids. Perhaps smoking only becomes a risk factor when there are other risks present. It is not a neat, simple problem.

The question is, would a national policy that lowered the lipid levels of the population change coronary heart disease risks? It is unfair and inaccurate to describe supporters of that policy as evangelists. I would not describe members of the COMA committee as evangelists. Whatever their individual characteristics, as a committee they were sobre, careful and deliberate on making reasoned scientific judgements. It is not a case of 
evangelism versus science. It's a case of one scientific opinion versus another. The people on the COMA committee, and on the British Cardiac Society committee on prevention, acted as careful, reasoned scientific people. The searchers of truth are presented by Professor Mitchell as an embattled minority against the majority that has hopped on to the dietary-fat bandwagon. There is gratification in seeing oneself as a lonely battler for truth, but I am not sure it is an accurate portrayal of the current state of affairs. Those of us who actually have to deal with cardiologists and the medical profession in general, don't feel we are in the majority if we say we ought to be doing something about prevention. We feel like the embattled minority.

Professor Mitchell implied that relying on observational data might lead one to conclude that potato eating causes tuberculosis. This is amusing but misleading. One can make a causal judgement based on non-trial data, as in the case of smoking, and can come up with a reasoned policy. In my humble opinion, the committees did not all get it wrong, and that the recommendation to change the national diet may work in terms of preventing coronary disease. While I would not for a moment suggest one-to-one causality, it's certainly true that those countries that have had major reductions in coronary mortality, have been countries where there has been more activity about prevention. It is just possible there may be a causal connection.

Chairman: Dr Rifkind, do you want to add anything and to plunge into the fray?

$B$. M. Rifkind: Obviously I think the totality of the evidence points to a different conclusion than the one drawn by Professor Mitchell. To turn to the varieties of evidence that I briefly summarized this morning and to characterize them as an evangelistic view is alarming. We have animal evidence, population-based evidence and some clinical trial information and I think the coherence and the congruity of the evidence is strong.

So far as the issue of preventing death, that's a new one for me, and is a philosophical issue for doctors and all the members of the community to debate. I can't, in the face of coronary disease being our and your major cause of death and disability, whilst accounting for a considerable proportion of your total health-care cost, stand back and talk about whether a country has seven times the coronary disease rate of some other country or whether a country's rate is rising, as being of no concern of ours! I find that a curious pastime and it's a dangerous one for physicians to take.

Costs have been mentioned and I don't want to be superficial about them. Of course there will be costs in attending to the major cause of death and disability in our community, but there has been, I think, some exaggeration of the cost. The cost of a cholesterol check is measured in pence. A more comprehensive lipid evaluation is nearer to $f 10$ but to measure cholesterol is as cheap a measurement as you will find in the clinical chemistry spectrum. If one asks merely that one should measure cholesterol just as one readily measures blood pressure in the routine care of a patient, this would be a little bit more expensive but not much more than measuring blood pressure alone. I do not think that should be a major concern.

Professor Mitchell mentioned the findings from the Dupont study for the socioeconomic relationship. I would like to turn that on its head, as it was in the Dupont report. The Dupont survey indicated that there had been a considerable drop in cardiovascular deaths and disability in the Dupont employees and that that drop had occurred to a much greater extent in the upper socio-economic groups of the executives than in the Dupont blue-collar employees. For me that's just another signal that the practice of the upper socio-economic groups of lower dietary fat intake, slightly greater exercise levels, lower body-weight, and lower levels of blood pressure and cholesterol are contributing to the benefits that the Dupont executives are seeing. This should be a stimulus for us to conduct a preventative approach. 
I find it difficult to believe at this time that we are still debating a causal role for cholesterol in coronary disease. Yes, the prospective and epidemiological studies speak but don't speak totally to the issue. We have a paradigm in familial hypercholesterolaemia. This monogenic disorder results in a raised level of cholesterol in children in the absence of hypertension, smoking, obesity and diabetes and leads to cholesterol accumulation and atherosclerotic lesions. This, as Bill Roberts has characterized at the National Institute of Health, is no different in its basic characteristics from the atherosclerosis of much more elderly sufferers of atherosclerosis. Such children seldom survive the first or second decade.

Let me deal with the MRFIT study. I think it is showing some wisdom after the event to say you would have expected the $5 \%$ fall in cholesterol in the referred care group, compared with the $7 \%$ fall in the special intervention group. That was not expected in the late 1960 s and early 1970 s when that trial was initiated. We can perhaps use the information for a different argument. One might have argued that cholesterol levels are not amenable to change in populations; but the referred care group in the MRFIT study showed that cholesterol does change when populations are sufficiently convinced that such a change is desirable. As Professor Marmot said, the net outcome in MRFIT was that there was only a $2 \%$ difference in the cholesterol levels of the two groups and that a clinical trial, as far as cholesterol-lowering is concerned, did not exist in MRFIT. The only other data in MRFIT that are relevant is a further analysis showing that if you examine the degree of cholesterol lowering in the MRFIT subjects you find that it also correlates with a reduction in coronary heart disease risk. It is weak evidence and I do not want to offer it as a major observation but I think that MRFIT should be seen for what it is and for what it is not.

Finally, I want to reiterate, the question of total mortality. None of the trials has had a sufficient sample size to address the issue of total mortality, and the causes of the increased non-coronary mortality in the various trials have been heterogenous. I doubt if any consistent plausible understanding of total mortality really exists at the present time.

B. Lewis: To Professor Mitchell I would say that no trial has yet been designed or conducted to examine the question of whether a reduction in serum cholesterol modifies total mortality. I know of no such trial. I think it would be impossible to conduct a trial which is sufficiently large. He seems to have ignored the evidence of benefit from nineteen of the twenty-one published trials in which a reduction of cholesterol has been produced by drug therapy or diet. This is the sort of end-point which the trials were statistically designed to identify and yet he has expounded on the alleged harm done by reduction of plasma cholesterol to total mortality which is the sort of end-point which no trial has the statistical power to address. I do not understand the rather selective use of these trials in his talk.

Secondly, I would say that coronary heart disease is caused by atherosclerosis. $\mathrm{He}$ is quite right that it is not the only cause. It is a necessary cause, however, and thrombotic occlusion of coronary arteries occurs on a basis almost always of atherosclerosis; it is a necessary cause but it is not of course a sufficient one. There is evidence from animal models that raising the serum cholesterol causes atherosclerosis and thrombotic occlusion of coronary arteries. Tom Clarkson has published very convincing photographs of coronary heart disease in rhesus monkeys so treated.

Risk factors are not necessarily causes but they may be so. I think the trials of cholesterol reduction indicate a causal effect, so do the animal studies and the few human studies of atherosclerosis regression. He talks about extrapolating from trials to the population as a whole and I would like to ask him whether he wishes to conduct separately, trials each costing 150 million dollars for blue-collar workers, seeing that the 
LRCCT trial was addressing white-collar workers? Does he want non-whites, seeing as the trial had whites in it? Does he want those with red hair, or left-handers, those with green eyes and freckles; do we have no ability at all to extrapolate if the extrapolation is plausible?

To Professor James, I would only like to clarify the position of the European Atherosclerosis Society on population policy. He clearly has not read the policy of the European Atherosclerosis Society, for which he may be forgiven, because it is still in the word processor, but it does fully endorse everything that I believe Professor James stands for. It fully endorses public health policy for prevention of coronary heart disease. What little it adds to that is perhaps to give very firm proposals on how to implement such a policy, but it also gives reasons for believing that this is not sufficient to reduce coronary heart disease as low in the population as we would like. For that reason it does advocate that there should be gradual ascertainment of hypercholesterolaemia in the population using exactly the same methods as we have used for recognizing hypertension.

$M$. J. Gibney: Suppose the entire audience were persuaded to vote along the lines of Professor James, we could then embark on a policy to implement NACNE. Let us assume we were to achieve that. Let us then ask ourselves what would the impact be. Based on what we know, we would expect a reduction in serum cholesterol and we would expect a drop in body-weight and blood pressure. What worries me is the loose use of the words 'prevent and reduce'. Both Professor Lewis and Professor Marmot have in the last 10 minutes used the terms 'prevent coronary heart disease'. What exactly is meant by prevention? Is it not possible for the non-nutritionists involved to come up with some sort of meaningful morbidity and mortality goals other than food guidelines so that we know exactly what level of heart disease we're going to be content with, or are we only going to be content when there is no coronary heart disease?

T. A. B. Sanders: The first point relates to Professor Mitchell's criticism of trials. I would agree with him that the trials in men have shown really little benefit with regard to prevention of coronary heart disease but we have to take into account that these were in middle-aged men. This may be a little bit too late as atherosclerosis starts in early adult life, perhaps even earlier in the teens. Therefore the stage at which any intervention occurs is obviously very important. Looking at men of 65 years in the Los Angeles Veteran trial, I think one's not surprised that you don't really get much effect and that is something we will have to bear in mind.

The other point is 'how good is compliance?' We all know from giving people advice about weight loss. You tell people to go on a diet but they don't stick to the diet. This can be a major problem.

If we do look at some extreme dietary groups, living in Western countries, I can think of Seventh Day Adventists, segregated into meat-eating ones, vegetarian ones and vegan ones; their blood cholesterol levels are quite segregated as well. You find that the meat-eating and vegetarian ones have quite high blood cholesterol levels and the vegan ones very low. None of the groups smokes or drinks and there is an amazingly different incidence of coronary heart disease between those groups, the vegans having very low levels of coronary heart disease. When you have groups that do comply strictly to diet and are strongly motivated, you do seem to get effects and I think one of the reasons there has been confusion in dietary trials is: (1) that the intervention has been too late and (2) that people do not really comply with the diets.

For example, people go to a doctor and he gives them a diet sheet. They say they are following the diet but they do their normal thing. We saw this yesterday in one of the abstracts from Ireland, that patients were given advice by their doctors but did not follow it. 
$R$. Braude: I wish to react to Professor Lewis's statement on use and misuse of statistics. Throughout the 2 days we have seen a lot of extrapolations, and $I$ have been appalled by the misuse of statistics. Ronald Fisher would turn in his grave if he witnessed what has been said at this meeting.

If you see that straight lines are fitted to variables whose errors are much higher than the relevant differences under discussion, it makes one wonder about the validity of the evidence presented. The word 'significantly' has been used often inappropriately and some contributors even drew definite conclusions which suited their arguments, after stating that the data presented were 'statistically not significant'. Fisher, who arbitrarily selected $20-1$ as a guidance to interpretation of biological differences warned that the 20-1 chance should not be used as meaningful when applied to most biological means. When applied to the arguments presented here, one should certainly avoid using this level of significance as meaningful for drawing conclusions for the human population. And one should also seriously consider whether it is right to advocate alterations of the diet for the whole population, when only a very small proportion would benefit from it.

Chairman: Thank you very much. That is a challenge.

$B$. Ratcliffe: Isn't it true that the national diet is changing anyway and so perhaps by making some policy decision, either through education or whatever, that the changes can be given at least some direction. It seems to me that there is a section of society whose dietary changes are being influenced largely by considerations of whether the food is convenient to buy and prepare. There is another section of society who are aware of the benefits and are perhaps changing their nutritional intake to alter their health but they do not know where to get the right kind of advice or understanding of what might be the correct food to eat. Often people take advice from magazines, newspaper articles, or popular television programmes where people are giving advice who are not professionally qualified. There is nothing wrong with the old advice of a little moderation in all things, but the general public needs to know what is moderate. What is a moderate intake of fat, what is a moderate intake of dietary fibre? As far as the broader aspects of cardiovascular disease and other diseases are concerned, there is no great danger in recommending to people that they have a reasonable intake of dietary fibre and that they have a reasonable intake of fat because it may have benefits not only in terms of cardiovascular disease but also in terms of risk of colon cancer and in their general state of health.

Chairman: You are going back to the 'prudent' diet.

G. Gillett: Dr Galton gave us a talk about genetic factors. Now we accept genetic factors in diabetes and we are often screcning for affected subjects. Is there any reason why this should not be done for coronary heart disease and for hypercholesterolaemia?

D. J. Galton: This is just being started with a look at the familial segregation of coronary heart disease to try and work out what are the relevant factors. One interesting point that this raises is the great amount of population diversity with regard to genetic factors even in the UK population, making it quite difficult to make blanket rules for everyone. I think you have got to take everything at an individual level rather than at a population level.

M. Ashwell: I think that I will take up a previous point and make a few points about education and nutrition. There are good and bad academics in terms of the way they will actually help journalists to write articles, with some who remain silent and won't help journalists write a middle-of-the-road article at all. There are some academics who are very good and who are very helpful. There are some journalists who will go to a press reception of a pressure group and return with press handouts. Because they have to get their article in by 4 o'clock that afternoon they will simply write the line of the press 
handout and will not research their line properly. This is why the press is full of sensationalism and why the press as a whole is not interested in middle-of-the-road nutrition because to them it's rather boring. I would like to suggest that there should be more tolerance between academics and journalists, which might achieve more middle-ofthe-road nutrition education to the public.

J. Dibney: There seems to be two assumptions which are very often made and have certainly been made here today, without being challenged, which I would like to challenge. One is that a diet which is lower in saturated fat and higher in fibre is bound to be awful and is some kind of infliction on people. This seems to rest on the idea that the current diet has some kind of saintly merit and that somehow it has been arrived at carefully. In fact it has been arrived at in a very ad hoc way and under pressure, particularly from the food industry in terms of promotion and advertising and from the policy makers in terms of agricultural policy. It is important not to imagine that the sort of status quo has some kind of magic about it.

The other assumption, which I have heard before, is this idea that old age is bound to be absolutely horrendous and awful and it's much better to die in later middle age. I know that we have a lot of very distinguished older members of the society who are very active and I would put it to them, do they wish that they had 'smoked heavily throughout life and done all sorts of reckless things in order to ensure that they were dead before this point?'

$M$. Oliver: One of the difficulties that we are in and one of the reasons why this debate is taking place is the excessive and extravagant attitudes and claims of some of our colleagues in the United States. We should not mince our words about this. I have written an article earlier in the year which I headed 'propaganda, promises, problems and prospects in coronary heart disease'*. There has been excessive propaganda, for what reasons I am not clear, and promises that lowering cholesterol will, not might, lower coronary heart disease. While I agree in terms of very high cholesterol levels, because appropriate trials have been done, I simply cannot take the bald statement that lowering cholesterol will lower coronary heart disease. I cannot see the purpose of this propaganda. It may stem from the fact that in their training and in their psychology doctors want to try to 'do good', and 'doing good' means doing something, or from a political need in the USA to justify the huge expenditure of public money on clinical trials in coronary heart disease. Whereas in fact doing nothing may often do more good. Thus, in those who are at the lower end of the cholesterol distribution doing nothing might do more good than doing something, partly because of the harm that excess focusing on cholesterol levels can do and partly because we do not yet know how safe it is to lower cholesterol levels to very low levels. We should identify that we may be too emotionally involved in this and stand back from it and recognize that there are a great many unknowns. We should be cautious, rather humble and try to learn more. These are not popular qualities with health educationalists. For example, what is the explanation of the fact that in the twenty-six WHO countries, which have had coronary mortality statistics recorded over the last 10 years with any reliability, the female coronary mortality has gone down in every single one of these while in men it has gone up in some and down in others? What is the explanation of the differences in mortality in the two sexes? There are many other inconsistencies. Let us be humble and go on trying to ask questions and collect more data.

D. J. Galton: For better or worse the effect of this propaganda has generated a large amount of anxiety in the general population and anyone who treats affected patients will know they come demanding something. You cannot quote literature, political policy *Oliver, M. (1986). Circulation 73, 1-9. 
documents or trials at them. I think for better or worse you have to take the evidence as it stands that there may be some benefit in reducing blood cholesterol and advise them accordingly.

$E$. Wheeler: Professor Oliver has just mentioned one group that we have been waiting to hear about: that is women. I want to pick on Dr Braude's question about what proportion of the population are being involuntarily affected by some of the measures which we are hearing about. I am concerned about the impact of the sweeping measures that are being proposed, particularly on women in the families of men who may be considered at risk. We have a large female population who may not require or even respond to dietary modification, but who in practice have it imposed upon them. I would like to know what the speakers think about this. I have not seen anything about the upper quartile of cholesterol in women, although we have heard all about men from Dr Rifkind.

The other group that I am concerned about is people on low incomes. I know that some groups in the UK have done work on the costs of NACNE-type diets.

Chairman: You asked a factual question about the upper quartile cholesterol in women. I don't know whether any member of the group would respond to that.

B. Rifkind: There are studies of cholesterol distributions in women and there are prospective data in women. Simply speaking, cholesterol is a risk factor for coronary heart disease in women as it is in men, but the coronary disease tends to occur about 10-15 years later in women compared with men. I would think that the upper quartile accounts for more or less the same proportion of the total coronary disease burden in women as it does for men but at a considerably older age.

Chairman: Is it meant that at the same level of serum cholesterol women are likely to develop coronaries 10 years later than men?

$M$. Oliver: Probably yes -10 years later or even more. The data are not nearly so sound as for men. There is no equivalent Framingham-type study or MRFIT data equivalent in women. It is an important question because more than half of the adults in the country are women and we should be very cautious before we apply exactly the same recommendations to women as for men. Whilst it is true that coronary heart disease occurs about 10 years later, there are all sorts of complex problems concerning changes in lipids during the menopause which makes it really quite difficult to relate given plasma lipoprotein concentrations to coronary heart disease. One of the things that is evidently fairly clear, although it is scientifically not as sound as it should be, is that relatively high levels of cholesterol seem to be tolerated far better by women. For example, if you take severe familial hypercholesterolaemia, there are plenty of accounts of women who are living into their $60 \mathrm{~s}$ and $70 \mathrm{~s}$ with very high cholesterol levels and with poor LDL receptor response. Now we need to look at those more. Why do women appear to tolerate high cholesterol levels better than men?

Chairman: That is a very important question, particularly since probably two-thirds of this audience are women.

$R$. Cotterill: Can I make two points. The first that has not been mentioned at all, is the evidence from the Framingham study that the correlation between blood cholesterol and coronary risk varies substantially with age. It does seem to be important that in men over the age of 55 the line is flat, and one has the difficulty of what advice one should give to individuals of different ages. This brings me to my other point which concerns the question of whether there should be a national policy. It would be very much easier to decide if those who advocated it, gave us a little clearer idea of what they think the national policy should be. I think there is a fundamental gulf between a policy of information and that of enforcement. 
Chairman: I am now going to ask Professor James to respond to the important points put against his case, and particularly to that last question, and to deal with the question put by Dr Braude.

W. P. T. James: Dr Braude has put this question to me before about the use of correlation analysis. I think that none of us who are involved, are doing what I think you believe we are doing, in suggesting that a correlation automatically implies causation. One displays correlations as only part of a whole facet of information and then try and piece the whole thing together. So I am a little confused as to what you are objecting to in precise terms. We are very conscious of the fact that if no one wanted to go for a precise policy, it would be much better to have a sophisticated scheme which took into account not only our crude understanding but also our analysis of the probability of it being effective.

I suppose one reason why one is advocating a national policy that affects women as well as men, notwithstanding the paucity of data in women, is the fact that those people who get involved in attempting to suggest to people ways by which they might change their diet, recognize that it is a bizarre proposition to have one policy for the under $5 \mathrm{~s}$, another for men and a different diet for women. Also if you have an elderly member in your household then surely one should not interfere there. One can imagine the most exquisitely complicated culinary schemes if one is involved in that. What this simply illustrates is the appalling crude nature of what we are doing at the moment. It would be very nice, in my terms, if we could readily identify the low-risk groups, have a clear understanding of which people we could go for, how to identify them and then on a practical basis engage the appropriate journalists to help people to understand what to do. But I think that is a counsel of perfection. I personally believe that the COMA policy was a pretty good one given the people who were on the panel, including myself. It was a consensus decision. If we are going to talk to the public, we have to engage on that concensus process and not try and give ourselves ego trips by showing that we know much better than the rest of the world. I think we do have a responsibility to try to distinguish what we are doing. The terrible danger of putting across the consensus message is that when it proves to need changing, we will not actually have educated the public to a sufficient level of sophistication for them to recognize that we are going through a process of changing policy. Unfortunately we still have a lot of people who love to make chaos and will tell everybody that nobody can make up their minds; that in my terms is probably counter-productive. I think what we have got to do is to develop a policy where we can give a whole range of dietary options and then prepare people for the fact that it could change, probably, I hope, in small ways.

Chairman: Thank you all for being so patient. I might just end with a quotation from Edmund Burke: 'He did the most harm, who for fear of doing harm, did nothing'. 